\title{
Association between IL- I8/I8R gene polymorphisms and coronary artery disease: influence of IL- I8/I8R genetic variants on cytokine expression
}

This article was published in the following Dove Press journal: Journal of Inflammation Research

\section{Vadim Mitrokhin' \\ Alexey Nikitin ${ }^{2}$ \\ Olga Brovkina ${ }^{2}$ \\ Dmitry Khodyrev² \\ Alexander Zotov ${ }^{2}$ \\ Nikita Vachrushev' \\ Dmitry Dragunov 3 \\ Andrey Shim' \\ Mitko Mladenov ${ }^{1,4}$ \\ Andre Kamkin'}

'Department of Fundamental and Applied Physiology, Russian National Research Medical University, Moscow, Russia; ${ }^{2}$ Federal Scientific Clinical Center for Specialized Types of Medical Assistance and Medical Technologies for the Federal Medical and Biological Agency, Moscow, Russia; ${ }^{3}$ Scientific Research Institute of Healthcare Organization and Medical Management, Moscow, Russia; ${ }^{4}$ Faculty of Natural Sciences and Mathematics, Institute of Biology, "Ss. Cyril and Methodius" University, Skopje, Macedonia
Correspondence: Mitko Mladenov Department of Fundamental and Applied Physiology, Russian National Research Medical University, Ostrovitjanova I, Moscow I I7997, Russia

$\mathrm{Tel} / \mathrm{fax}+7095$ । 3 | 4863

Emailm.mitko@gmail.com
Purpose: The present study investigated the influence of IL-18/18R genetic variants on cytokine expression in patients with stable coronary artery disease (CAD).

Materials and methods: The polymorphisms rs1946518, rs187238, rs326, rs1169288, and rs183130 were determined in patients with and without CAD. Circulating cytokine levels were measured immunologically.

Results: The rs 1946518-GG genotype shows higher IL-18 concentration in the group with CAD, but still not significant. The TG genotype from rs 1946518 in carriers with CAD showed a significant decrease in relation to the pro-inflammatory cytokines IL-6, IL-8, and IL-18. The decreases of IL-6 and IL- 8 were also specific for rs 187238 CAD carriers with the GC genotype. The CAD carriers with the AA genotype from rs326 in the IL-18R gene showed significant increase in IL- 8 and IL-18 in comparison with those without CAD. Regarding rs 1169288 from the IL-18R gene, IL- 8 showed a $\mathrm{T}$ allele-dependent increase. In the last rs183130 polymorphism of the IL-18R gene, the pro-inflammatory onset showed a C allele-dependent disease-associated decrease in IL-8 CC and IL-6 CT carriers. In contrast, the CAD CT carriers in relation to IL-8 showed significant increase. Conclusions: Most of the IL-18/18R single-nucleotide polymorphisms were mainly associated with pro-inflammatory cytokines. It is surmised that these associations between some proinflammatory cytokines (mainly IL-8) and some IL-18R genotypes in the subjects with CAD from this study are most likely based on inflammatory-induced upregulation of IL-18R expression. Keywords: Russian patients, inflammation, gene polymorphisms, IL-18/IL-18R genetic variants, inflammation, cytokines

\section{Introduction}

IL-18 acts synergistically with IL-12 and induces the production of interferon- $\gamma($ IFN- $\gamma$ ) in T cells, subsets of macrophages, and natural killer cells. ${ }^{1-3}$ Despite its costimulatory activities in the production of IFN- $\gamma$, IL-18 is able to activate the natural factor kappa $\mathrm{B}(\mathrm{NF}-\kappa \mathrm{B})^{4}$ and induce production of pro-inflammatory cytokines and chemokines, such as a tumor necrosis factor- $\alpha$ (TNF- $\alpha)$, IL-1 $\beta$, IL-6, and IL-8. ${ }^{5,6}$ This ability to induce pro-inflammatory cytokines classifies IL-18 as a command molecule in the coordination of the immune responses. ${ }^{7}$

It was recently established that IL-18 acts via a complex receptor, which possesses a binding chain (IL-18R $\alpha$ ) and a signaling chain (IL-18R $\beta$ ) ${ }^{8-10}$ Even with conflicting results, the elevated circulatory IL-18 has been associated with complex settings in diabetes type 2, atherosclerotic lesions, hypertension, metabolic syndrome, and 
coronary artery disease $(\mathrm{CAD}) \cdot{ }^{8-10}$ Additional factors that may significantly influence the levels of IL-18 are genetic polymorphisms. ${ }^{11}$ Besides the existing disparity in the genetic data, some IL-18 genetic variants may influence the risk of CAD, modify the expression of IL-18, and change the circulating levels of IL-18. ${ }^{12}$

In order to extend the existing knowledge regarding the importance of the IL-18/IL-18R gene variants, their distribution in the Russian population with CAD, the influence on IL-18 levels, and their association with other cytokines, we employed different statistical models. Taking into account the role of IL-18 in the process of inflammation, we assumed that IL-18/IL-18R genetic polymorphisms could be reflected in varying levels of different cytokines, which would be differently expressed depending on the onset of the disease. Finding an association between these cytokines and some of the tagged single-nucleotide polymorphisms (SNPs) could have a significant impact on the further establishment of new clinical targets for earlier determination of the risk of CAD development.

This single-center study was conducted at the Federal Scientific Clinical Center for Specialized Types of Medical Assistance and Medical Technologies, Moscow (FSCCSTMAMT) Cohort. Two SNPs in the IL-18 gene (rs1946518 and rs 187238) and three SNPs (rs326, rs1169288, and rs183130) in the IL-18R gene were selected.

\section{Materials and methods}

\section{Ethical considerations}

This study was approved by the Ethics Committee from the FSCCSTMAMT (protocol no. 003/2016) and conducted in accordance with the Declaration of Helsinki. All participants were adequately informed about the project prior to their inclusion in the study, and they completed the registration form providing written informed consent containing details concerning age, gender, hypertension, diabetes mellitus, hypercholesterolemia, and smoking habits.

\section{Sample size computation}

Calculation of sample size and baseline characteristics of the patients are given in details in our previous study. ${ }^{13}$ Briefly, the district demographic data from the 2012 Census, with a population of 507,000 inhabitants were taken for sample size computation. Assuming a 10\% margin of error and a maximum prevalence of $50 \%$, with a $95 \% \mathrm{CI}$, a sample minimum was defined at 126 participants (cases). Taking into account the possible loss of $10 \%$, the final sample size was estimated at 176 participants (cases). Controls were defined according to the approximate number of cases, including 116 participants. Statdisk Version 8.4 was used to calculate the sample size. ${ }^{14}$

\section{Patients}

One hundred seventy-six CAD and 116 control angiographically characterized patients were included in this study. All analyses were conducted on patients admitted to FSCCSTMAMT. All participants in the study were of Russian origin. The control subjects were confirmed to be free of $\mathrm{CAD}$ and other cardiovascular diseases, liver and end-stage renal diseases, thyroid disease, immune system diseases, malignant tumors, and serious infections. Hypertension, diabetes mellitus, and hypercholesterolemia were classified according to the same criteria as in our previous study. ${ }^{13}$ The basic criteria for inclusion in the group with CAD was $\geq 50 \%$ stenosis in at least one coronary artery (for details see our previous study by Mitrokhin et $\mathrm{al}^{13}$ ). The basic criteria for exclusion from the group with CAD were coronary artery bypass graft intervention, history of percutaneous coronary intervention, congenital heart disease, severe heart failure, cardiomyopathy, rheumatic valvular heart disease, end-stage renal and liver diseases, thyroid disease, immune system diseases, malignant tumor, connective tissue disease, and serious infections. Both groups were composed of patients suspected of having CAD, but those without determined CAD were taken as controls. All patients included in the study did not take statins.

\section{Blood collection}

Blood samples were collected under fasting conditions between 8.00 and 10.00 a.m. and prepared for routine analyses. Plasma was prepared within 1 hour at room temperature by centrifugation at $2000 \times g$ for $15 \mathrm{~min}$. The plasma was stored at $-80^{\circ} \mathrm{C}$ until analyses were performed. An electrochemical-luminescence immunoassay (pro-BNP Elec-sys; Roche Diagnostics GmbH, Mannheim, Germany) was used for the N-terminal pro B-type natriuretic peptide (Nt-proBNP) determination.

\section{Clinical data}

The clinical data of the control and CAD subjects, including measurements of glucose, blood pressure (BP), total cholesterol (TC), triglyceride (Tg), low-density lipoprotein cholesterol (LDL-c), high-density lipoprotein cholesterol, Nt-proBNP, IFN- $\gamma$, bilirubin, and fatty acid-binding protein (FABP), were compiled from the medical records.

\section{Circulating concentrations of IL-2, IL-4, IL-6, IL-8, IL-I8, IL-I $\beta$, and IL-I 0}

The newly developed enzyme-linked immunosorbent assay, for quantitative analysis of IL-2, IL-4, IL-6, IL-8, IL-18, IL-1 $\beta$, and IL-10 levels, from Bender Med-Systems was 
used to analyze these proteins, and IL-10 in the serum. The assay's limit of detection was about $1.9 \mathrm{pg} / \mathrm{mL}$ for IL-2, 0.5 $\mathrm{pg} / \mathrm{mL}$ for IL-4, $0.94 \mathrm{pg} / \mathrm{mL}$ for IL-6, $2.2 \mathrm{pg} / \mathrm{mL}$ for IL-8, $2.2 \mathrm{pg} / \mathrm{mL}$ for IL-18, $1.2 \mathrm{pg} / \mathrm{mL}$ for IL- $1 \beta$, and $1.1 \mathrm{pg} / \mathrm{mL}$ for IL-10. Inter- and intra-assay coefficients of variation were $7.4 \%$ and $6.3 \%$ for IL-2, $9.2 \%$ and $8.6 \%$ for IL-4, $6.4 \%$ and $4.6 \%$ for IL-6, $7.8 \%$ and $6.9 \%$ for IL-8, $6.6 \%$ and $7.6 \%$ for IL- $18,6.8 \%$, and $4.7 \%$ for IL- $1 \beta$, and $8.3 \%$ and $7.5 \%$ for IL- 10 .

\section{Genotyping of IL- I8 and IL-I8R}

Genotyping of all the studied SNPs was conducted through real-time polymerase chain reaction (PCR) allelic discrimination with TaqMan probes. The probes and primer designs were conducted utilizing UGENE software (version 1.14; http://ugene.unipro.ru) and Oligo Analyzer software (version 1.0.3). Gene sequences were obtained from the National Center for Biotechnology (http://www.ncbi.nlm.nih.gov). The genotype and allele frequencies were calculated via direct counting. The estimation of the adherence of the genotype distribution to the Hardy-Weinberg equilibrium was conducted by the De-Finetti program Exact Test (https://ihg.gsf. de/cgi-bin/hw/hwa1.pl). The case-control status was provided by insertion of 26 blinded quality control samples in order to assure genotyping procedure validation; the blinded samples' concordance was $>99 \%$. Probes, primers, and genotyping assays are available upon request.

\section{Statistical methods}

Continuous variables are expressed as mean \pm SD. Categorical variables are expressed as frequencies and percentages $(\%)$. Group comparisons were performed with the Kruskal-Wallis test and the Mann-Whitney test for continuous data and Fisher's exact test with Yates correction followed by Bonferroni correction for categorical data. The normality distribution for each variable was checked. Log transformation was used in the case of significantly skewed variables. A two-tailed probability test of 0.05 or less was considered statistically significant. Associations between the SNPs and the serum cytokine levels were assessed by analysis of variance and post-hoc Tukey's test. The multivariate logistic model was employed to analyze the association between the studied SNPs and the risk of CAD by determining the odds ratio (OR) and its corresponding 95\% CI. Homozygotes of the most frequent genotypes were used as a reference group in both genes. All statistical analyses were performed using SPSS 17.0 software (SPSS, Chicago, IL, USA).

\section{Results}

\section{Patients' baseline characteristics}

Baseline characteristics of the control and CAD patients are shown in Table 1. The details about the baseline characteristics of the studied groups of patients are given in our previous study. ${ }^{13}$ Briefly, the group with CAD had a statistically significant variation in gender and circulatory level of Tgs in comparison with the control group. The low level of significant difference in all studied parameters between the two examined groups is obvious from Table 1 . We believe that the basic reason for such a low level of statistical significance lies in the overlap of the baseline characteristics (mainly, diabetes mellitus and hypertension) between the two examined groups of patients.

\section{Genotypes and allele frequencies of the IL-I 8 and IL-I8R SNPs}

The genotype distributions for all SNPs were in the HardyWeinberg equilibrium. In rs1946518, 84 (29.16\%) were GG homozygotes, 48 (16.66\%) were TT homozygotes, while $156(54.16 \%)$ were TG heterozygotes, and 6 subjects remained undetermined. The allele frequency of the $\mathrm{G}$ allele in rs 1946518 was 0.853 in the whole study population. In rs187238, 52 (46.42\%) were GG homozygotes, 48 (42.86\%) were GC heterozygotes, and 12 (10.71\%) were CC homozygotes, while 4 subjects remained undetermined in the group without CAD. The same SNP in the group with CAD had a similar genotype distribution, with 86 (48.31\%) GG homozygotes, 78 (43.82\%) GC heterozygotes, and $14 \mathrm{CC}$ (7.87\%) homozygotes. The allele frequency of the $\mathrm{G}$ allele in rs 187238 was 0.721 throughout the whole study population.

Table I Baseline characteristics of the study population

\begin{tabular}{|c|c|c|c|}
\hline Variables & $\begin{array}{l}\text { Controls } \\
(n=116)\end{array}$ & $\begin{array}{l}\text { Patients } \\
(n=176)\end{array}$ & $p$-value \\
\hline Age (years) & $74.94 \pm 7.43$ & $70.97 \pm 12.94$ & 0.723 \\
\hline Gender (M/F) & $33 / 83$ & $98 / 78$ & 0.000 \\
\hline BMI $\left(\mathrm{kg} / \mathrm{m}^{2}\right)$ & $30.33 \pm 6.09$ & $31.05 \pm 2.69$ & 0.612 \\
\hline Smoking (yes/no) & $7 / 109$ & $11 / 165$ & 0.735 \\
\hline Hypertension (yes/no) & $63 / 53$ & $81 / 95$ & 0.101 \\
\hline $\begin{array}{l}\text { Type } 2 \text { diabetes mellitus } \\
\text { (yes } / \text { no) }\end{array}$ & $40 / 76$ & $60 / 116$ & 0.746 \\
\hline $\mathrm{TC}(\mathrm{mmol} / \mathrm{L})$ & $5.379 \pm 1.46$ & $5.20 \pm 1.08$ & 0.384 \\
\hline $\mathrm{Tg}(\mathrm{mmol} / \mathrm{L})$ & $1.705 \pm 1.91$ & $1.4 I \pm 0.69$ & 0.021 \\
\hline LDL-c (mmol/L) & $3.294 \pm 1.24$ & $3.02 \pm 0.98$ & 0.336 \\
\hline HDL-c (mmol/L) & $1.64 \pm 0.73$ & $1.58 \pm 0.52$ & 0.171 \\
\hline $\mathrm{CA}$ & $2.28 \pm 0.4 I$ & $2.38 \pm 0.35$ & 0.368 \\
\hline
\end{tabular}

Notes: $p$-values $<0.05$ are shown in bold. Data are presented as mean \pm standard deviation.

Abbreviations: BMI, body mass index; CA, coefficient of atherogenicity; HDL-c, high-density lipoprotein cholesterol; LDL-c, low-density lipoprotein cholesterol; TC, total cholesterol; Tg, triglyceride. 
The genotypes and allele frequency distribution of IL$18 \mathrm{R}$ polymorphisms were as follows. In rs326 from the group without CAD, 76 (66.66\%) were AA homozygotes, 32 (28.07\%) were GA heterozygotes, and 6 (5.26\%) were GG homozygotes, with 2 undetermined subjects. The genotype distribution with the same SNP from the CAD patients was 102 (57.95\%) AA homozygotes, 68 (38.63\%) GA heterozygotes, and $6(3.4 \%) \mathrm{GG}$ homozygotes. The allele frequency of the A allele in rs326 was 0.636 in the whole study population. The genotype distribution of rs 1169288 in the group without CAD was 34 (29.31\%) TT homozygotes, 52 (44.83\%) TG heterozygotes, and 30 (25.86\%) GG homozygote, with a
T allele frequency of 0.966 . In the group with $\mathrm{CAD}$, the distribution was 68 (38.2\%) TT homozygotes, $92(51.69 \%) \mathrm{TG}$ heterozygotes, and 18 (10.11\%) GG homozygotes, with a T allele frequency of 0.780 . In the CAD patients, rs $183130 \mathrm{had}$ 78 (44.32\%) CC homozygotes, 70 (39.77\%) CT heterozygotes, and 28 (15.91\%) TT homozygotes; the group without CAD contained 54 (49.09\%) CC homozygotes, 42 (38.18\%) CT heterozygotes, and 14 (12.73\%) TT homozygotes. The allele frequency of the $\mathrm{C}$ allele in rs 183130 was 0.733 in the group without $\mathrm{CAD}$ and 0.779 in the CAD subjects (Table 2 ).

In general, both groups of patients had a similar genotype distribution for all tagged polymorphisms. The genotype

Table 2 Genotype distributions of different SNPs in patients with and without CAD and their association with risk of CAD development

\begin{tabular}{|c|c|c|c|c|c|}
\hline Variables & Patients without CAD & Patients with CAD & OR (95\% Cl) & p-value & pc-value \\
\hline IL-I 8 & $n=116(\%)$ & $n=176(\%)$ & & & \\
\hline \multicolumn{6}{|l|}{ rs I9465 I 8} \\
\hline GG & $28(24.56)$ & $56(32.18)$ & 1.0 & & \\
\hline TG & $66(57.89)$ & $90(51.72)$ & $1.47(0.84-2.55)$ & 0.174 & ns \\
\hline TT & $20(17.54)$ & $28(16.09)$ & $1.03(0.53-1.98)$ & 0.937 & ns \\
\hline \multicolumn{6}{|l|}{ Allele } \\
\hline G & $122(53.5 \mid)$ & $202(58.05)$ & 1.0 & & \\
\hline $\mathrm{T}$ & $106(46.49)$ & $146(41.95)$ & $1.32(0.95-1.85)$ & 0.102 & ns \\
\hline \multicolumn{6}{|l|}{ rs187238 } \\
\hline GG & $52(46.43)$ & $86(48.31)$ & 1.0 & & \\
\hline GC & $48(42.85)$ & $76(43.18)$ & $1.01(0.6 \mathrm{I}-1.59)$ & 0.914 & ns \\
\hline $\mathrm{CC}$ & $12(10.71)$ & $14(7.87)$ & $0.72(0.3 \mid-1.68)$ & 0.444 & ns \\
\hline \multicolumn{6}{|l|}{ Allele } \\
\hline G & $152(67.86)$ & $250(70.22)$ & 1.0 & 0.538 & ns \\
\hline C & $72(32.14)$ & $102(28.97)$ & $1.09(0.76-1.58)$ & 0.538 & ns \\
\hline \multicolumn{6}{|l|}{ IL-I 8R } \\
\hline \multicolumn{6}{|l|}{ rs326 } \\
\hline $\mathrm{AA}$ & $76(66.66)$ & $102(57.95)$ & 1.0 & & \\
\hline GA & $32(28.07)$ & $68(38.64)$ & $0.63(0.38-1.06)$ & 0.079 & ns \\
\hline GG & $6(5.26)$ & $6(3.4 I)$ & $0.47(0.14-1.57)$ & 0.213 & ns \\
\hline \multicolumn{6}{|l|}{ Allele } \\
\hline A & $184(80.70)$ & $272(77.27)$ & 1.0 & & \\
\hline G & $44(19.30)$ & $80(22.73)$ & $0.8 \mathrm{I}(0.54-\mathrm{I} .23)$ & 0.325 & ns \\
\hline \multicolumn{6}{|l|}{ rsII69288 } \\
\hline TT & $34(29.31)$ & $68(38.20)$ & 1.0 & & \\
\hline TG & $52(44.83)$ & $92(51.69)$ & $1.13(0.66-1.93)$ & 0.652 & ns \\
\hline GG & $30(25.86)$ & $16(9.09)$ & $0.33(0.16-0.66)$ & 0.001 & ns \\
\hline \multicolumn{6}{|l|}{ Allele } \\
\hline $\mathrm{T}$ & $120(51.72)$ & $220(63.00)$ & 1.0 & & \\
\hline G & I I 2 (48.28) & $132(37.00)$ & $1.59(1.13-2.23)$ & 0.007 & ns \\
\hline \multicolumn{6}{|l|}{ rs183130 } \\
\hline $\mathrm{CC}$ & 54 (49.09) & 78 (44.32) & 1.0 & & \\
\hline $\mathrm{CT}$ & $42(38.18)$ & $70(39.77)$ & $0.87(0.52-1.45)$ & 0.587 & ns \\
\hline TT & $14(12.73)$ & $28(15.91)$ & $1.20(0.57-2.53)$ & 0.632 & ns \\
\hline \multicolumn{6}{|l|}{ Allele } \\
\hline C & $150(68.18)$ & $226(64.20)$ & 1.0 & 0.329 & ns \\
\hline $\mathrm{T}$ & $70(31.82)$ & $126(35.80)$ & $0.84(0.59-1.20)$ & & \\
\hline
\end{tabular}

Notes: $p$-values, Fisher's exact test with Yates's correction; pc-value, after Bonferroni correction. Bold values denote $p<0.05$.

Abbreviations: CAD, coronary artery disease; IL, interleukin; ns, nonsignificant; OR, odds ratio; SNPs, single-nucleotide polymorphisms. 
distribution in the group without $\mathrm{CAD}$ was $20.4 \%$ for $\mathrm{GG}$, $20.8 \%$ for GT, $12 \%$ for CC, $8.4 \%$ for GC, $13.4 \%$ for AA, $5.6 \%$ for GA, $12.01 \%$ for TT, and $7.4 \%$ for CT. The genotype distribution in the group with CAD was $18.82 \%$ for GG, $20.63 \%$ for GT, $10.43 \%$ for CC, $8.84 \%$ for GC, $11.56 \%$ for AA, $7.71 \%$ for GA, $14.06 \%$ for TT, and $7.94 \%$ for CT.

\section{Plasma IL- I 8 concentrations in relation to IL-I 8 genotypes}

In the whole CAD population, there were no significant changes in the IL-18 plasma level according to all tested genotypes. Actually, the rs $1946518 \mathrm{G}$ allele was weakly associated with the lower IL-18 levels as compared with the rs1946518 TT genotype ( $p=0.065$; medians ( 25 th, 75 th percentiles): $118.27(65,157)$ versus $139.51(98,171) \mathrm{pg} / \mathrm{mL}$, respectively, but still statistically not significant $(p>0.1$; Table 3).

\section{Statistical differences between the patients with CAD and controls, irrespective of their genotypes}

The IL-1 $\beta$, IL-8, and FABP in the group with CAD were significantly higher as compared with the control group $(p=0.015,0.000,0.000)$ (Table 4$)$.

Table 3 Plasma levels of IL-I8 in relation to the IL-I8 polymorphisms of the total CAD population

\begin{tabular}{|c|c|c|c|}
\hline SNP & Genotype & IL-I $8(\mathrm{pg} / \mathrm{mL})^{\mathrm{a}}$ & $p$-value ${ }^{b}$ \\
\hline \multicolumn{4}{|c|}{ rs1946518 } \\
\hline & GG & II $8.27(65,157)$ & $>0.065$ \\
\hline & TG & $86.21(56,113)$ & \\
\hline & TT & $|39.5|(98,17 \mid)$ & \\
\hline \multicolumn{4}{|c|}{ rsI 87238} \\
\hline & GG & $148.15(72,180)$ & $>0.363$ \\
\hline & GC & $95.35(53,123)$ & \\
\hline & $\mathrm{CC}$ & $106.45(68,149)$ & \\
\hline \multicolumn{4}{|c|}{ rs326 } \\
\hline & AA & $94.33(57,155)$ & $>0.631$ \\
\hline & GA & $104.31(74,146)$ & \\
\hline & GG & I49.52 (| |9, I80) & \\
\hline \multicolumn{4}{|c|}{ rs|l69288 } \\
\hline & TT & $106.24(78,154)$ & $>0.453$ \\
\hline & TG & $90.16(56,135)$ & \\
\hline & GG & $104.39(53,177)$ & \\
\hline \multicolumn{4}{|c|}{ rs I 83130} \\
\hline & $\mathrm{CC}$ & $101.49(61,123)$ & $>0.565$ \\
\hline & $\mathrm{CT}$ & $104.24(58,164)$ & \\
\hline & TT & $98.50(64,200)$ & \\
\hline
\end{tabular}

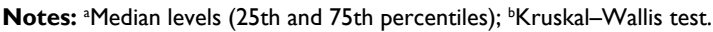
Abbreviations: CAD, coronary artery disease; IL, interleukin; SNP, singlenucleotide polymorphisms.
Cytokine relationships between different genotypes from IL-I8/IL-I8R SNPs associated with CAD

The rs 1946518-GG genotype showed a significantly higher IL-8 concentration in the group with CAD ( $p=0.0019$, Table 5A). The TG genotype from rs1946518 in the carriers with CAD showed a significant decrease in relation to pro-inflammatory cytokines $(p=0.0052$ for IL- $6, p=0.0002$ for IL-8, and $p=0.0029$ for IL-18). Directional decrease ( $p=0.0362$ for IL- 6 and $p=0.0089$ for IL-8, Table $5 \mathrm{~A}-\mathrm{E}$ ) was also specific for the GC genotype from the rs $187238 \mathrm{CAD}$ carriers. Notably, in the same group of patients, IL-1 $\beta$ showed a statistically significant increase ( $p=0.0077$, Table $5 B)$. The increase was also seen in IL- 8 in the GG genotype from the same SNP $(p=0.0003)$. The CAD carriers with AA genotype from rs326 in the IL-18R gene showed significant increases in IL-8 and IL- 18 in comparison with controls ( $p=0.0001$ and 0.043 , respectively, Table $5 \mathrm{C}$ ). In the patients with the same polymorphism of the same gene with the GA genotype, IFN- $\alpha$ was significantly increased $(p=0.0236$ ), while IL-6 was significantly decreased $(p=0.0349)$ in comparison with controls. Interestingly, in rs1169288 in the IL-18R gene, IL-8 showed a T allele-dependent increase. Actually, regarding rs1169288, circulatory IL-8 was significantly increased in the TG and TT CAD carriers ( $p=0.0057$ and 0.0039 , respectively), while in the CAD GG carriers it was decreased $(p=0.0053)$. The CAD-associated decrease in the pro-inflammatory markers was also $\mathrm{T}$ allele dependent. It

Table 4 Statistical differences between the controls and patients, irrespective of their genotypes

\begin{tabular}{ll}
\hline CAD vs Control & p-value \\
\hline IL-2 & 0.874 \\
IL-4 & 0.196 \\
IL-6 & 0.485 \\
IL-8 & 0.000 \\
IL-I8 & 0.404 \\
IL-I $\beta$ & 0.015 \\
IL-I0 & 0.137 \\
IFN- $\alpha$ & 0.154 \\
Bilirubin & 0.077 \\
IFN- $\gamma$ & 0.066 \\
NT-proBNP & 0.058 \\
Myoglobin & 0.744 \\
FABP & 0.000 \\
Troponin & 0.309 \\
\hline
\end{tabular}

Note: $p$-values $<0.05$ are shown in bold.

Abbreviations: CAD, coronary artery disease; FABP, fatty acid-binding protein; IFN- $\gamma$, interferon gamma; IL, interleukin; NT-proBNP, N-terminal pro B-type natriuretic peptide. 
Table 5 Relationships between genotypes from IL-I8/IL-I8R SNPs between patients with and without CAD in relation to some inflammatory and clinically CAD-associated parameters

\begin{tabular}{llll}
\hline (A) & \multicolumn{3}{l}{ IL-I8 rs I 9465 I 8 } \\
\cline { 2 - 4 } & GG & TG & TT \\
\hline IL-2 & 0.224 & 0.054 & 0.805 \\
IL-4 & 0.688 & 0.054 & 0.999 \\
IL-6 & 0.670 & $\mathbf{0 . 0 0 5}$ & 0.151 \\
IL-8 & $\mathbf{0 . 0 0 I}$ & $\mathbf{0 . 0 0 0}$ & 0.087 \\
IL-I8 & 0.939 & $\mathbf{0 . 0 0 2}$ & 0.627 \\
IL-Ib & 0.476 & 0.481 & 0.056 \\
IL-I0 & 0.431 & 0.082 & 0.853 \\
IFN- $\alpha$ & 0.307 & 0.110 & 0.429 \\
Bilirubin & 0.551 & 0.421 & 0.422 \\
IFN- $\gamma$ & 0.555 & 0.629 & 0.854 \\
NT-proBNP & 0.781 & 0.795 & 0.900 \\
Myoglobin & 0.922 & 0.172 & 0.372 \\
FABP & 0.843 & 0.875 & 0.895 \\
Troponin & 0.482 & 0.112 & 0.201 \\
\hline
\end{tabular}

\begin{tabular}{llll}
\hline (B) IL-18 rs 187238 & \\
\hline CC & GC & GG
\end{tabular}

\begin{tabular}{llll} 
& CC & GC & GG \\
\hline IL-2 & 0.763 & 0.124 & 0.321 \\
IL-4 & 0.725 & 0.354 & 0.898 \\
IL-6 & 0.384 & $\mathbf{0 . 0 3 6}$ & 0.123 \\
IL-8 & 0.130 & $\mathbf{0 . 0 0 8}$ & $\mathbf{0 . 0 0 0}$ \\
IL-I8 & 0.365 & 0.083 & 0.189 \\
IL-Ib & 0.053 & $\mathbf{0 . 0 0 7}$ & 0.195 \\
IL-I0 & 0.935 & 0.513 & 0.591 \\
IFN- $\alpha$ & 0.504 & 0.331 & 0.421 \\
Bilirubin & 0.511 & 0.442 & 0.682 \\
IFN- $\gamma$ & 0.492 & 0.677 & 0.428 \\
NT-proBNP & 0.245 & 0.284 & 0.199 \\
Myoglobin & 0.516 & 0.403 & 0.367 \\
FABP & 0.273 & 0.108 & 0.126 \\
Troponin & 0.367 & 0.197 & 0.241 \\
\hline
\end{tabular}

\begin{tabular}{llll}
\hline (C) & \multicolumn{2}{l}{ IL-I 8R rs326 } & \\
\cline { 2 - 4 } & AA & GA & GG \\
\hline IL-2 & 0.309 & 0.183 & 0.218 \\
IL-4 & 0.841 & 0.311 & 0.240 \\
IL-6 & 0.121 & $\mathbf{0 . 0 3 5}$ & 0.949 \\
IL-8 & $\mathbf{0 . 0 0 0}$ & $\mathbf{0 . 0 0 0}$ & 0.533 \\
IL-I8 & $\mathbf{0 . 0 4 2}$ & 0.246 & 0.801 \\
IL-Ib & 0.151 & 0.914 & 0.460 \\
IL-I0 & 0.983 & 0.054 & 0.460 \\
IFN- $\alpha$ & 0.239 & $\mathbf{0 . 0 2 3}$ & 0.113 \\
Bilirubin & 0.441 & 0.712 & 0.505 \\
IFN- $\gamma$ & 0.262 & 0.361 & $0.4 I 1$ \\
NT-proBNP & 0.482 & 0.381 & 0.272 \\
Myoglobin & 0.503 & 0.455 & 0.377 \\
FABP & 0.706 & 0.664 & 0.519 \\
Troponin & 0.222 & 0.199 & 0.348 \\
\hline
\end{tabular}

\begin{tabular}{llll}
\hline (D) & \multicolumn{4}{l}{ IL-I 8R rs I I69288 } \\
\cline { 2 - 4 } & GG & TG & TT \\
\hline IL-2 & 0.150 & 0.17 I & 0.423 \\
IL-4 & 0.460 & 0.399 & 0.818 \\
IL-6 & 0.378 & 0.166 & $\mathbf{0 . 0 I I}$ \\
\hline & & & (Continued)
\end{tabular}

Table 5 (Continued)

\begin{tabular}{|c|c|c|c|}
\hline \multirow[t]{2}{*}{ (D) } & \multicolumn{3}{|c|}{ IL-I IR rs I I 69288} \\
\hline & GG & TG & TT \\
\hline IL-8 & 0.005 & 0.005 & 0.003 \\
\hline IL-18 & 0.560 & 0.042 & 0.403 \\
\hline IL-Ib & 0.682 & 0.305 & 0.516 \\
\hline IL-I0 & 0.488 & 0.860 & 0.404 \\
\hline IFN- $\alpha$ & 0.987 & 0.181 & 0.269 \\
\hline Bilirubin & 0.892 & 0.799 & $0.46 I$ \\
\hline IFN- $\gamma$ & 0.991 & 0.891 & 0.628 \\
\hline NT-proBNP & 0.659 & 0.722 & 0.502 \\
\hline Myoglobin & 0.369 & 0.428 & 0.273 \\
\hline FABP & 0.264 & 0.353 & 0.449 \\
\hline Troponin & 0.577 & 0.664 & 0.482 \\
\hline \multirow[t]{2}{*}{ (E) } & \multicolumn{3}{|c|}{ IL-I8R rs I $83 \mid 30$} \\
\hline & CC & CT & TT \\
\hline IL-2 & 0.093 & 0.053 & 0.550 \\
\hline IL-4 & 0.251 & 0.626 & 0.100 \\
\hline IL-6 & 0.131 & 0.011 & 0.950 \\
\hline IL-8 & 0.002 & 0.001 & 0.081 \\
\hline IL-I8 & 0.077 & 0.116 & 0.625 \\
\hline IL-Ib & 0.198 & $0.74 I$ & 0.470 \\
\hline IL-IO & 0.784 & 0.227 & 0.973 \\
\hline IFN- $\alpha$ & 0.229 & 0.081 & 0.303 \\
\hline Bilirubin & 0.144 & 0.104 & 0.416 \\
\hline IFN- $\gamma$ & 0.201 & 0.098 & 0.229 \\
\hline NT-proBNP & 0.163 & 0.125 & 0.317 \\
\hline Myoglobin & 0.277 & 0.209 & 0.169 \\
\hline FABP & 0.101 & 0.146 & 0.337 \\
\hline Troponin & 0.305 & $0.37 \mathrm{I}$ & 0.178 \\
\hline
\end{tabular}

was observed that there was a decrease in IL-18 in the TG heterozygotes $(p=0.042)$ and in IL- 6 in the TT homozygotes $(p=0.012$, Table $5 \mathrm{D})$. In the last rs 183130 polymorphism in the IL-18R gene, the pro-inflammatory onset showed C allele-dependent disease-associated decrease ( $p=0.0023$ for IL-8 CC and $p=0.0111$ for IL-6 CT carriers). In contrast, diseased CT carriers in relation to IL-8 showed a significant increase ( $p=0.0016$, Table 5E).

In general, the IL-18/IL-18R genotype biomarker interaction between the patients with and without diagnosed CAD were completely different. The results from this study have confirmed involvement of IL-18 in the inflammation and interaction with other inflammatory mediators in the onset of CAD. ${ }^{15,21,22}$ The association between IL-18 SNPs (rs1946518 and rs187238) and IL-18R SNPs (rs326, rs1169288, and rs 183130) and particular circulatory cytokine concentrations were shown and can be taken as a basis for further investigation of inflammation/CAD interactions. 


\section{Logistic regression model for CAD}

As in our previous study, ${ }^{13}$ applied logistic regression model revealed only gender $(\mathrm{OR}=1.88)$ and $\mathrm{Tg}(\mathrm{OR}=2.61)$ to be the strongest determinants of CAD (Table 6). None of the studied SNP genotypes determine susceptibility to CAD.

\section{Discussion}

This research revealed that the $G$ allele from the rs 1169288 carriers is associated with CAD onset. This study's findings are similar to those of Morita et $\mathrm{al}^{11}$ who reported a significant association between the common coding variants of rs1 169288 and multiple cardiovascular risk phenotypes in Euro-American adults. Based on a reported variety of associations between IL-18 SNPs and CAD, ${ }^{11}$ in this study, we examined the associations between IL-18/IL-18R SNPs and circulating levels of different cytokines in the Russian population. In this direction, different epidemiologic studies have revealed that specific SNPs in the different genes such as IL-6, ${ }^{13,16-18} \mathrm{IL}-17,{ }^{19} \mathrm{IL}-18,{ }^{20}$ and IL-23 are associated with the development of CAD. ${ }^{21-27}$ In the present study, a significant association between the CAD GG carriers of rs 1946518 and rs187238 SNPs and the higher circulating IL-8 levels was shown. In contrast, their respective heterozygote carriers (TG and GC) showed a significant association with lower IL-6 and IL-8 levels. Also, a lower IL-18 concentration was associated with rs 1946518-TG, while rs 187238 -GC was associated with increased IL-1 $\beta$. On the other hand, an increased release of IL-18 associated with the $\mathrm{G}$ allele of rs187238-GC in the circulating leukocytes from the patients with CAD was reported. ${ }^{28}$ Also, Giedraitis et al, ${ }^{5}$ by studying the transcriptional activity of the IL-18 in stimulated human HeLa 229 cells transfected with the promoter region of IL-18, reported an increased transcriptional activity in stimulated $\mathrm{C}$ and $\mathrm{G}$ alleles (rs1946518 and rs 187238 , respectively). ${ }^{5}$ This result is contradictory to the obtained lower IL-18 circulating levels from our study and also to the previously reported results for lower gene expression from lymphoblastic cell lines. ${ }^{28}$ Assuming that circulating leukocytes may not be the main source contributing to circulating IL-18, the reported increase may not be of biological significance. In relation to

Table 6 Logistic regression analysis for patients

\begin{tabular}{lll}
\hline Variables & p-value & OR $(\mathbf{9 5 \%} \mathbf{~ C l})$ \\
\hline Gender & $\mathbf{0 . 0 2 9}$ & $1.88(1.15-2.92)$ \\
$\mathrm{Tg}$ & $\mathbf{0 . 0 3 7}$ & $2.61(1.12-3.58)$ \\
\hline
\end{tabular}

Note: $p$-values $<0.05$ are shown in bold. Abbreviations: $\mathrm{OR}$, odds ratio; $\mathrm{Tg}$, triglyceride. our study, given that both the studied groups have the same body mass index (but we do not know if they also have the same adipose tissue distribution [IL-18 may be produced by adipose tissue]), we assume that IL-18 could be the same, just for this reason. Furthermore, Tiret et $\mathrm{al}^{29}$ examined five IL-18 SNPs (rs1946519, rs5744292, rs4937100, rs360717, and rs549908) in 1288 patients with CAD and found only rs 5744292 (described there as A+183G in exon 6) to be significantly associated with IL-18 serum levels. On the other hand, such an association was not the case for rs187238 SNP (described as $-137 \mathrm{G}>\mathrm{C}$ ) within the IL-18 gene in black South-African women with cardiovascular disease. ${ }^{30}$ Based on these studies and based on the fact that some population studies ${ }^{11}$ find associations between type 2 diabetes, hypertension, and specific IL-18 SNPs with the susceptibility to CAD,${ }^{31}$ it seems that the described discrepancy in our study is the result of its specificity based on the selection of the examined groups. Actually, the fact that none of the studied SNPs genotypes determine susceptibility to CAD according to the applied regression model in our study represents another corroboration of the described discrepancy. Certainly, partial roles of particular genotypes from all tagged SNP in such a complex mechanism could be determined, but because of the huge discrepancies in the distribution of the studied genotypes, this analysis was not carried out.

In the CAD subjects from this study, IL-18R rs 326 AA genotype was associated with significantly higher circulating levels of IL-18 and IL-8. This supports the increased AA raising effect during inflammation. A similar exaggeration was determined for the $A G$ genotype in the same SNP, expressed through IL-6, IL-8, and IFN- $\alpha$. In the same direction, IL-18R rs1169288 was especially interesting due to its influence on the IL- 8 concentration in all different genotype carriers (GG, GT, and TT). GT and TT carriers had by a decreased pro-inflammatory milieu (IL-18 for GT and IL-6 for TT). Identical genotype-cytokine relationships were registered between rs $1169288 \mathrm{TT}$ and rs 183130 CT genotype carriers, which reflected the dominant role of the $\mathrm{C}$ allele. These associations between some pro-inflammatory cytokines (mainly IL-8) and some IL-18R genotypes in the CAD subjects from this study are plausibly based on the fact that the pro-inflammatory mediators induced upregulation of the IL-18R expression. The study by Krásná et $\mathrm{al}^{7}$ at the level of renal and respiratory epithelial cell lines showed that both the cell lines upregulated IL-18R mRNA and IL-18R membrane expression in response to pro-inflammatory cytokines. In addition, 
in the same study, the function of IL-18R was confirmed by the induction of IL- 8 released from the epithelial cells in response to a recombinant IL-18. Theoretically, it seems that in the case of pro-inflammatory cytokines, similar disease/genotype interactions can be found in different genotype carriers. ${ }^{32}$

In addition to the abovementioned associations between SNPs and various cytokines in patients with CAD, the logistic regression model in our study outlined Tg as a possible indicator for CAD. These results are in agreement with the study of Holvoet et $\mathrm{al}^{33}$ who reported a positive association between rising Tg and $\mathrm{CAD}$ in different ethnic groups. The main role of Tg in lipid metabolism is not directly atherogenic, but Tg is taken as an important indicator of the risk of CAD due to its association with the atherogenic remnant particles. ${ }^{34,35}$ The nonsignificant coefficient of atherogenicity from our study represents another confirmation of the nonatherogenic nature of the significantly increased $\mathrm{Tg}$.

In conclusion, by examining the inflammatory and antiinflammatory cytokines in patients with $\mathrm{CAD}$, it was revealed that IL-1 $\beta$ and IL-8, like pro-inflammatory cytokines, are significantly higher in patients with CAD. It was noted that most IL-18 SNPs were somehow associated with pro-inflammatory cytokine changes. It seems that determined differences between the controls and the group with CAD within the same genotypes could be explained by the fact that the existing polymorphisms in IL-18R may modify the response to IL-18, and in turn the production of inflammatory mediators, only when the inflammatory background still exists.

\section{Limitations}

The cross-sectional nature of the study is one of the basic limitations. The prospective prediction of the effects of IL-18/ IL-18R genotypes upon CAD development is impossible at this stage. Another limitation stems from the fact that FSCCSTMAMT is a third-level clinical institution in the Russian healthcare system. Hence, recruitment of pure control subjects was almost impossible (all visitors to the center are already suspected or classified as diseased patients from the first or second level). This was the basic reason why we had overlap in the baseline characteristics between the two studied groups of patients. The strength of this study is in its homogeneous population consisting of patients from the same race and nationality.

\section{Acknowledgment}

This work was supported by the Russian Science Foundation (grant no. 16-14-10372).

\section{Author contributions}

All authors contributed toward data analysis, drafting and revising the paper and agree to be accountable for all aspects of the work.

\section{Disclosure}

The authors report no conflicts of interest in this work.

\section{References}

1. Dinarello CA. Interleukin-18, a proinflammatory cytokine. Eur Cytokine Netw. 2000;11(3):483-486.

2. Evans J, Collins M, Jennings C, et al. The association of interleukin-18 genotype and serum levels with metabolic risk factors for cardiovascular disease. Eur J Endocrinol. 2007:157(5):633-640.

3. Fantuzzi G, Reed DA, Dinarello CA. IL-12-induced IFN-gamma is dependent on caspase-1 processing of the IL-18 precursor. J Clin Invest. 1999;104(6):761-767.

4. Galimudi KR, Spurthi MK, Padala C, et al. Interleukin 6(-174G/ C) variant and its circulating levels in coronary artery disease patients and their first degree relatives. Inflammation. 2014;37(2): 314-321.

5. Giedraitis V, He B, Huang WX, Hillert J. Cloning and mutation analysis of the human IL-18 promoter: a possible role of polymorphisms in expression regulation. J Neuroimmunol. 2001;112(1-2):146-152.

6. Huang $\mathrm{H}$, Zeng $\mathrm{Z}$, Zhang L, et al. The association of interleukin-16 gene polymorphisms with susceptibility of coronary artery disease. Clin Biochem. 2013;46(3):241-244.

7. Krásná E, Kolesár L, Slavcev A, et al. IL-18 receptor expression on epithelial cells is upregulated by TNF-alpha. Inflammation. 2005;29(1): 33-37.

8. Mallat Z, Corbaz A, Scoazec A, et al. Expression of interleukin-18 in human atherosclerotic plaques and relation to plaque instability. Circulation. 2001;104(14):1598-1603.

9. Mallat Z, Heymes C, Corbaz A, et al. Evidence for altered interleukin (IL)-18 pathway in human heart failure. FASEB J. 2004;18(14): 1752-1754.

10. Matsumoto S, Tsuji-Takayama K, Aizawa Y, et al. Interleukin-18 activated NF- $\kappa \mathrm{B}$ in murine T helper type 1 cells. Biochem Biophys Res Commun. 1997;234(2):454-457.

11. Morita K, Saruwatari J, Tanaka T, et al. Associations between the common HNF1A gene variant p. I27L (rs11,69,288) and risk of type 2 diabetes mellitus are influenced by weight. Diabetes Metab. 2015;41(1): 91-94.

12. Munder M, Mallo M, Eichmann K, Modolell M. Murine macrophages secrete interferon gamma upon combined stimulation with interleukin (IL)-12 and IL-18: a novel pathway of autocrine macrophage activation. J Exp Med. 1998;187(12):2103-2108.

13. Mitrokhin V, Nikitin A, Brovkina O, et al. Association between interleukin-6/6R gene polymorphisms and coronary artery disease in Russian population: influence of interleukin-6/6R gene polymorphisms on inflammatory markers. J Inflamm Res. 2017;10:151-160.

14. Triola MF. Statdisk (version 8.4) for Elementary Statistics. Rio de Janeiro. LTC. 2003;410.

15. Okamura H, Tsutsi H, Komatsu T, et al. Cloning of a new cytokine that induces IFN-gamma production by T cells. Nature. 1995;378(6552): 88-91.

16. Shim LA, Aksyonov AA, Mitrokhin VM, et al. Serum interleukin-6: association with circulating cytokine serum levels in patients with sinus arrhythmia and patients with coronary artery disease. Cell Immunol. 2016;310:178-183.

17. Kazanski V, Mitrokhin VM, Mladenov MI, Kamkin AG. Cytokine effects on mechano-induced electrical activity in atrial myocardium. Immunol Invest. 2017;46(1):22-37. 
18. Mitrokhin VM, Mladenov MI, Kamkin AG. Effects of interleukin-6 on the bio-electric activity of rat atrial tissue under normal conditions and during gradual stretching. Immunobiology. 2015;220(9):1107-1112.

19. Ovchinnikov RS, Mitrokhin VM, Mladenov MI. Effects of interleukin17A on the bioelectric activity of rat atrial myocardium under normal conditions and during gradual stretching. Cytokine. 2015;76(2): 561-565.

20. Vm M, Al S, Aa A, et al. Circulating interleukin-18: association with IL-8, IL-10 and VEGF serum levels in patients with and without heart rhythm disorders. Int J Cardiol. 2016;215:105-109.

21. Okamura H, Tsutsui H, Kashiwamura S, Yoshimoto T, Nakanishi K. Interleukin-18: a novel cytokine that augments both innate and acquired immunity. Adv Immunol. 1998;70:281-312.

22. Olee T, Hashimoto S, Quach J, Lotz M. IL-18 is produced by articular chondrocytes and induces proinflammatory and catabolic responses. J Immunol. 1999;162(2):1096-1100.

23. Opstad TB, Pettersen AA, Arnesen H, Seljeflot I. Circulating levels of IL-18 are significantly influenced by the IL-18 $+183 \mathrm{~A} / \mathrm{G}$ polymorphism in coronary artery disease patients with diabetes type 2 and the metabolic syndrome: an observational study. Cardiovasc Diabetol. 2011;10:110.

24. Dziedziejko V, Kurzawski M, et al. Association between IL-18 gene polymorphisms and the release of interleukin-18 from stimulated peripheral blood mononuclear cells. Biotechnol Biotechnol Eq. 2012;26(1):2987-2990.

25. Belenkova Y, Karetnikova V, Diachenko A, et al. Association of inflammatory markers and poor outcome in diabetic patients presenting with ST segment elevation myocardial infarction. J Inflamm Res. 2015;8: 107-116.

26. Shadrina A, Voronina E, Zolotukhin I, Filipenko M. Allele and genotype frequencies of polymorphisms in cytokine genes in ethnic Russian individuals from Moscow, Russia. Hum Immunol. 2017;78(2): 190-191.
27. Mouridsen MR, Nielsen OW, Carlsen CM, et al. High-sensitivity $\mathrm{C}$-reactive protein and exercise-induced changes in subjects suspected of coronary artery disease. J Inflamm Res. 2014;7:45-55.

28. Puren AJ, Fantuzzi G, Dinarello CA. Gene expression, synthesis, and secretion of interleukin 18 and interleukin 1beta are differentially regulated in human blood mononuclear cells and mouse spleen cells. Proc Nat Acad Sci USA. 1999;96(5):2256-2261.

29. Tiret L, Godefroy T, Lubos E, et al; Athero Gene Investigators. Genetic analysis of the interleukin-18 system highlights the role of the interleukin-18 gene in cardiovascular disease. Circulation. 2005;112(5): 643-650.

30. Evans J, Collins M, Jennings C, et al. The association of interleukin-18 genotype and serum levels with metabolic risk factors for cardiovascular disease. Eur J Endocrinol. 2007;157(5):633-640.

31. Zhang X, Pei F, Zhang M, et al. Interleukin-17A gene variants and risk of coronary artery disease: a large angiography-based study. Clin Chim Acta. 2011;412(3-4):327-331.

32. Zhang M, Cai ZR, Zhang B, et al. Functional polymorphisms in interleukin-23 receptor and susceptibility to coronary artery disease. DNA Cell Biol. 2014;33(12):891-897.

33. Holvoet P, Jenny NS, Schreiner PJ, Tracy RP, Jacobs DR; Multi-Ethnic Study of Atherosclerosis. Multi-Ethnic study of atherosclerosis. The relationship between oxidized LDL and other cardiovascular risk factors and subclinical CVD in different ethnic groups: the multi-ethnic study of Atherosclerosis (MESA). Atherosclerosis. 2007;194(1):245-252.

34. McLarty JL, Li J, Levick SP, Janicki JS. Estrogen modulates the influence of cardiac inflammatory cells on function of cardiac fibroblasts. J Inflamm Res. 2013;6:99-108.

35. Miller M, Stone NJ, Ballantyne C, et al; Council on Cardiovascular Nursing; Council on the Kidney in Cardiovascular Disease. Triglycerides and cardiovascular disease: a scientific statement from the American Heart Association. Circulation. 2011;123(20):2292-2333.
Journal of Inflammation Research

\section{Publish your work in this journal}

The Journal of Inflammation Research is an international, peer-reviewed open access journal that welcomes laboratory and clinical findings on the molecular basis, cell biology and pharmacology of inflammation including original research, reviews, symposium reports, hypothesis formation and commentaries on: acute/chronic inflammation; mediators of

\section{Dovepress}

inflammation; cellular processes; molecular mechanisms; pharmacology and novel anti-inflammatory drugs; clinical conditions involving inflammation. The manuscript management system is completely online and includes a very quick and fair peer-review system. Visit http://www.dove press.com/testimonials.php to read real quotes from published authors. 